\title{
Prevalencia de trastornos de la personalidad y su asociación con la recaída post-tratamiento en pacientes policonsumidores de sustancias en una unidad de tratamiento residencial
}

\author{
Prevalence of personality disorders and its association with post- \\ treatment relatives in polymonsumident patients of substances in a \\ residential treatment unit
}
Daniela Nieto Preciado ${ }^{1}$, Beatriz Corona Figueroa ${ }^{2 *}$, Diana Ortiz Sánchez ${ }^{3}$, Humberto Venegas Peña ${ }^{4}$ y Rosa Meda Lara ${ }^{5}$

Universidad de Guadalajara

Recibido: $29-03-17$

Aceptado: $01-06-17$

\begin{abstract}
Resumen
Los trastornos de personalidad comórbidos al consumo de sustancias generan recaídas y deterioro funcional. La evaluación temprana permite formular objetivos terapéuticos adecuados. Objetivo: Establecer la frecuencia de recaída a cuatro meses de seguimiento en pacientes consumidores de sustancias con trastornos de personalidad. Método: Se utilizó la Structured Clinical Interview for DSM-IV Axis II Personality Disorders (SCID II) para evaluar trastornos de personalidad y examen de detección de sustancias en orina. Resultados: Se evaluaron 33 pacientes, y $36.4 \%$ presentaba trastornos de personalidad; el antisocial tuvo la mayor prevalencia. Los pacientes con trastornos de personalidad presentaron recaída en el consumo. Conclusión: Los tratamientos para consumo de sustancias deberán incluir intervenciones para trastornos de personalidad y otras comorbilidades para prolongar la abstinencia. Palabras clave: prevalencia, trastornos relacionados con sustancias, recaída, trastornos de personalidad, tratamiento residencial.
\end{abstract}

\footnotetext{
*Autor para correspondencia

1. Centro Universitario de Los Valles, Universidad de Guadalajara. Email: dcnietopreciado@gmail.com

2. Facultad de Psicología, Universidad Autónoma de Guadalajara. Centro Universitario de Ciencias de la Salud, Universidad de Guadalajara y Centro Universitario de Ciencias de la Salud, Universidad de Guadalajara. Email: beatriz.coronafi@outlook.com, beatriz.corona@edu.uag.mx

3. Centro Universitario de Los Valles, Universidad de Guadalajara. Email: diana.ortiz@profesores.valles.udg.mx

4. Facultad de Psicología, Universidad Autónoma de Guadalajara. email: alejandrovp77@gmail.com

5. Centro Universitario de Ciencias de la Salud, Universidad de Guadalajara. Email: rosa.meda@academicos.udg.mx
}

\footnotetext{
(C) Los autores. Este artículo es publicado por la Revista de Investigación en Psicología de la Facultad de Psicología, Universidad Nacional Mayor de San Marcos. Este es un artículo de acceso abierto, distribuido bajo los términos de la licencia Creative Commons Atribucion - No Comercia_Compartir Igual 4.0 Internacional. (http://creativecommons.org/licenses/by-nc-sa/4.0/) que permite el uso no comercial, distribución y reproducción en cualquier medio, siempre que la obra original sea debidamente citada.
} 


\begin{abstract}
Introduction: Personality disorders that are comorbid to substance use lead to relapse and functional deterioration. Early evaluation allows for the formulation of appropriate therapeutic goals. Objective: To establish the frequency of relapse to four months of follow-up in patients consuming substances with personality disorders. Method: The Structured Clinical Interview for DSM-IV Axis II Personality Disorders (SCID II) was used to evaluate personality disorders and urine substance screening to evaluate abstinence. Results: A total of 33 patients were evaluated, and $36.4 \%$ presented personality disorders; The antisocial disorder had the highest prevalence. Patients with personality disorders had relapse in consumption. Conclusion: Treatments for substance use should include interventions for personality disorders and other comorbidities to prolong substance withdrawal.

Keywords: prevalence, substance-related disorders, recurrence, personality disorders, drug treatment centers.
\end{abstract}

Los rasgos de personalidad son patrones persistentes de formas de percibir, relacionarse y pensar sobre el entorno y sobre uno mismo que se ponen de manifiesto en una amplia gama de contextos sociales y personales, se transforman en trastornos de la personalidad cuando son inflexibles y desadaptativos, omnipresentes, de inicio precoz, resistentes al cambio y cuando causan un deterioro funcional significativo o un malestar subjetivo (American Psychiatric Association, 2002; Esbec y Echeburúa, 2011).

Los individuos con trastorno de personalidad exhiben conductas rígidas y desadaptativas, que causan incapacidad funcional, tienden a crear círculos viciosos que perpetúan las dificultades y muestran baja estabilidad emocional en condiciones de estrés. Los rasgos de estos trastornos son evidentes desde la adolescencia y los primeros años de la edad adulta (Sánchez, 2003).

\title{
TRASTORNOS DE LA PERSONALIDAD Y CONSUMO DE SUSTANCIAS
}

La investigación ha mostrado que una gran cantidad de problemas que acompañan al consumo de sustancias, procede de patrones disfuncionales de conducta, que se mantienen en el tiempo con elevada estabilidad y pueden justificar, también en parte, tanto la persistencia de la conducta adictiva como la dificultad de manejo de los pacientes que los presentan (Pedrero, Puerta, Lagares y Sáenz, 2003).

El consumo de sustancias podría interpretarse como un intento fallido de relación. Los consumidores de sustancias son personas emocionalmente dependientes que buscan afecto, atención y aceptación pero sus reacciones impulsivas e imprevisibles terminan provocando rechazo. Tienden a aislarse socialmente por temor a involucrarse, les cuesta expresar y regular sus emociones de manera adecuada, controlar sus impulsos y establecer relaciones cercanas (Corona-Figueroa, Robles García, Meda-Lara, Fresán Orellana y Cruz-Maycott, 2015; Sánchez, Guisa, Cedillo y Pascual, 2002). 
La evaluación de los trastornos de personalidad es compleja por la diversidad de instrumentos de evaluación, criterios diagnósticos y clasificaciones. En nuestro país, una de cada cinco personas con un trastorno del Eje I presenta un trastorno de personalidad y casi la mitad de personas con un trastorno de personalidad padecen un trastorno del Eje I (Benget, Borges y Medina-Mora 2008). En sujetos con abuso o dependencia de sustancias, resulta aún más complejo el diagnóstico debido al tiempo de consumo, su nivel de funcionalidad o su grado de deterioro.

Una revisión de estudios realizados en relación a la comorbilidad de los trastornos de la personalidad con el alcoholismo, entre los años 1989 y 2002, muestra la presencia de por lo menos uno de estos trastornos en pacientes alcohólicos. La tasa de prevalencia encontrada oscila entre el 30 y el $80 \%$ dado que se emplearon diferentes instrumentos y metodologías para la evaluación y los trastornos asociados con mayor frecuencia a pacientes alcohólicos, fueron el antisocial, histriónico, paranoide y narcisista (Fernández-Montalvo y Landa, 2003).

En algunos estudios que muestran alta prevalencia de trastornos de personalidad (De Medina, Echeburúa y Aizpiri, 2008; Martínez, Graña y Trujillo, 2009; Martínez, Graña y Trujillo, 2011; Martínez, Albein, Lozano y Verdejo, 2014), donde el instrumento utilizado fue el Examen Internacional de los Trastornos de la Personalidad (IPDE), se encontró que en usuarios poli-consumidores de sustancias el trastorno histriónico, limítrofe y evitativo de la personalidad son los trastornos más frecuentes, en tanto que en los casos de usuarios consumidores de alcohol hubo predominio de los trastornos obsesivo-compulsivo y dependiente de la personalidad.

Por otra parte en algunos estudios en donde se utilizó el Inventario Clínico Multiaxial de Millon-II (MCMI-II) para la evaluación y diagnóstico de los trastornos de la personalidad en usuarios poli-consumidores (Pedrero, Ruiz, Lozoya, Rojo, Llanero y Puerta, 2013; López y Becoña, 2006; Fernández-Montalvo y Javier, 2004; Pedrero y Segura, 2003; Pedrero, Puerta, Lagares y Saez, 2003), se encontró mayor presencia de los trastornos histriónico, limítrofe, negativista y antisocial de la personalidad.

Resultados similares se han encontrado con la SCID II para la evaluación y diagnóstico de los trastornos de personalidad en usuarios de sustancias (Bricolo, Gomma, Bertani y Serpelloni, 2002), en donde los trastornos encontrados fueron limítrofe, antisocial, narcisista, paranoide y obsesivo-compulsivo.

\section{Consumo de sustancias, trastornos de la personalidad y evolución terapéutica}

La existencia de trastornos de personalidad en pacientes con tratamiento residencial o ambulatorio para rehabilitación por abuso o dependencia de sustancias se confirmó en el estudio realizado a 978 sujetos poli-consumidores en donde se 
encontró un trastorno o incluso varios, lo que sugiere mayor vulnerabilidad y riesgo de agravamiento en la evolución del paciente durante el tratamiento (De Medina et al., 2008; Herrero, 2004).

La falta de adherencia de los pacientes al tratamiento tiene diversas causas y es una de las problemáticas más graves en el ámbito de las drogodependencias. La mayoría de los abandonos ocurren en las primeras semanas del tratamiento. El consumo de drogas suele ir acompañado de otros trastornos y síntomas que pueden impedir la rehabilitación si no son identificados y tratados adecuadamente (García, Secades, Rodríguez, Rodríguez, Fernández-Hermida, Carballo et al., 2007).

La presencia de trastornos como el límite y el antisocial de la personalidad, seguidos de impulsividad y emociones adversas, representan un riesgo elevado de abandono del tratamiento (Martínez et al., 2014). Recientemente se ha encontrado que la presencia de trastornos de la personalidad en pacientes poli-consumidores se relaciona con autoengaño, negación, amnesia selectiva, proyección y pensamiento fantaseado, que se asocian positivamente con el craving y negativamente con la abstinencia (Martínez, Vilar, Becoña y Verdejo, 2016). La calidad de vida de los consumidores con trastorno de la personalidad es menor que la de aquellos sin el trastorno asociado y no acostumbran solicitar ayuda profesional, lo que impacta fuertemente su adherencia al tratamiento (Martínez, et al., 2011).

Dada la alta prevalencia de los trastornos de personalidad en los consumidores de sustancias y la alta tasa de abandono del tratamiento o recaídas en pacientes consumidores de sustancias con trastorno de la personalidad reportados por la literatura, el objetivo del presente estudio fue determinar la prevalencia de estos trastornos y su asociación con la recaída en el consumo a cuatro meses de su egreso del tratamiento. Se hipotetizó que aquellos pacientes que presentaran trastorno de la personalidad tendrían mayores probabilidades de recaer en el consumo durante el seguimiento que aquellos que no presentaran este diagnóstico.

\section{MÉTODO}

La Unidad de Tratamiento Residencial donde se trabajó atiende a pacientes provenientes principalmente de la República Mexicana, varones y mujeres a partir de los 14 años de edad, cuyo nivel de consumo sea Grado III o funcional y Grado IV o disfuncional, consumidores de una o varias sustancias legales o ilegales. El sistema de ingreso es abierto, todos los pacientes se incorporan en diferentes momentos y tienen fechas de ingreso y egreso distintas. El presente trabajo tiene un diseño transversal analítico.

\section{Sujetos}

Participaron en el estudio pacientes de nuevo ingreso a la unidad de tratamiento residencial, consumidores de sustancias y con primaria terminada. Para la 
selección, se excluyó a los pacientes que padecieran trastorno psicótico, deterioro cognitivo grave, que no estuvieran ubicados en tiempo y espacio, o que no hubieran terminado la primaria. Los pacientes se seleccionaron mediante muestro por conveniencia, tomando en cuenta los criterios de exclusión, su asignación para el tratamiento residencial de estancia media y su consentimiento para participar, por lo que la muestra definitiva fue de 33 pacientes.

\section{Variables}

Se consideró como variable independiente el diagnóstico de la SCID-II en sus categorías: sin diagnóstico, rasgos de la personalidad y trastorno de la personalidad. Como variable dependiente se consideró el resultado del seguimiento de los pacientes (inasistencia al examen, reporte vía telefónica o resultado del examen de detección de sustancias en orina).

\section{Procedimiento}

Los pacientes seleccionados aceptaron participar de forma confidencial y firmaron consentimiento informado para la aplicación del cuestionario, entrevista y examen de detección de sustancias en orina, mismo que se practicaría cuatro meses después de su egreso con la finalidad de constatar la abstinencia o el consumo. Este seguimiento se llevó a cabo mediante una llamada telefónica para conocer su estado actual y asignar una cita para su asistencia al examen. Si el paciente reportaba telefónicamente el consumo, se registraba este resultado e igualmente se le asignaba cita. En caso de no asistir, se contaba como resultado el reporte telefónico. Si no se le localizaba, esto se registraba como no asistencia.

\section{Instrumentos de evaluación y registro}

Entrevista inicial: Es el formato utilizado en las entrevistas de primera vez con todos los pacientes ingresados a la unidad de tratamiento residencial. Registra datos sociodemográficos como sexo, edad, escolaridad y empleo, así como motivo de consulta, patrón de consumo, sustancia de mayor impacto y diagnóstico específico de consumo de sustancias.

Entrevista Structured Clinical Interview for DSM-IV Axis II Personality Disorders (SCID II) :(First, Spitzer, Gibbon, \& Williams, 1997), es un instrumento utilizado para evaluar los 11 trastornos de personalidad del eje II del DSM-IV. Consiste en un cuestionario de 123 preguntas cerradas y una entrevista semiestructurada para corroborar si los rasgos reportados en el cuestionario son patológicos, persistentes y generalizados, en cuyo caso se les considera rasgos y con ellos se diagnostica de acuerdo a los criterios del DSM-IV para cada trastorno. La aplicación total del instrumento toma aproximadamente 60 minutos.

Examen de detección de sustancias en orina: La prueba utilizada es la Rapid Drug Screen (RDS), esta prueba es presuntiva y reporta información cualitativa, 
con un rango de confiabilidad y especificidad del $99 \%$ y es la única prueba con validez legal para confirmar y determinar resultados positivos. Los puntos de corte varían según la sustancia a medir: para Cocaína es de 300ng/ml; para Cannabis es 50ng/ml AMP $1000 \mathrm{ng} / \mathrm{ml}$, para Metanfetaminas 1,000 ng/,1 BZO $300 \mathrm{ng} / \mathrm{ml}$, para Opiáceos es de $300 \mathrm{ng} / \mathrm{ml}$ y para Barbitúricos $200 \mathrm{ng} / \mathrm{ml}$. (American Bio Medical Corporation, 2015).

\section{Análisis estadísticos}

Se empleó el paquete estadístico SPSS 22 y se obtuvieron frecuencias y porcentajes de variables sociodemográficas y de consumo. Dado que la hipótesis planteaba que aquellos pacientes con trastorno de la personalidad presentarían mayores probabilidades de recaída al seguimiento, el diagnóstico principal de $\sin$ diagnóstico, rasgos de la personalidad o trastorno de la personalidad según la SCID-II se utilizó como variable predictora y resultado del examen al seguimiento se planteó como variable de efecto. El diagnóstico de rasgos o trastorno de la personalidad tomó 3 categorías: 1) no presentó diagnóstico, 2) trastornos de la personalidad y 3) rasgos de la personalidad. La variable de los resultados del seguimiento tomó tres categorías: 1) inasistencia al examen, 2) reporte telefónico de consumo y 3) resultado positivo del examen. Debido al tamaño de la muestra, se practicó un análisis de tabla de contingencia para conocer la asociación entre el diagnóstico de rasgos o trastorno de personalidad y el resultado obtenido en el seguimiento, mediante pruebas de Chi Cuadrado, con un nivel de significancia de $\mathrm{p} \leq 0.05$.

\section{RESULTADOS}

La tabla 1 muestra los resultados obtenidos en las variables sociodemográficas. El $84.8 \%$ de los pacientes evaluados son hombres, el $15.2 \%$ son mujeres; reportaron estar casados $42.4 \%$, solteros $36.4 \%$, divorciados $18.2 \%$ y el $3 \%$ otro estado civil; en cuanto a la escolaridad, el $42.4 \%$ reportó tener el nivel básico, el $27.3 \%$ reportó haber llegado al bachillerato, el 30.3\% alcanzó el nivel superior. Respecto a la ocupación el $60.5 \%$ reportó estar desempleado, el $27.3 \%$ cuenta con empleo estable y $6.1 \%$ son estudiantes y realizan actividades del hogar respectivamente. La sustancia de mayor imparto en los pacientes es el alcohol 30.3\%, seguido de la mariguana $24.2 \%$, cristal $18.2 \%$; el consumo de cocaína se presentó en $15.2 \%$, con menor frecuencia el consumo de crack 9.1\% y los solventes con el 3\%. Dentro del Eje I el $75.7 \%$ de los pacientes presentó dependencia a varias sustancias y el $24.3 \%$ otros trastornos.

Los resultados de la entrevista SCID-II respecto al diagnóstico de trastornos de personalidad se muestran en la tabla 2; el $24.2 \%$ de los pacientes no presentó trastorno o rasgos; el $27.4 \%$ presentó trastorno antisocial de la personalidad, mientras que el trastorno obsesivo compulsivo, paranoide y limite se registraron en el 3\% de los pacientes respectivamente. En cuanto a los rasgos de personalidad, 
el $18.2 \%$ de los pacientes presentó rasgos del Grupo B, el 12.1\% presentó rasgos del grupo A y el 9.2\% presentó rasgos del Grupo C.

Finalmente, en la tabla 3 se describe el resultado del análisis de Chi cuadrado y se muestra la asociación entre el diagnóstico de trastorno de la personalidad y los resultados del examen de detección de sustancias en orina. Es importante mencionar que todos los pacientes concluyeron su tratamiento y de acuerdo a su fecha de egreso, se buscó contactarlos cuatro meses después para el seguimiento.

Tabla 1

Datos socio demográficos de la muestra ( $n=33)$

\begin{tabular}{|c|c|c|}
\hline Variable & Frecuencia & Porcentaje \\
\hline \multicolumn{3}{|l|}{ Edad } \\
\hline 14 a 19 años & 7 & $21.2 \%$ \\
\hline 20 a 39 años & 11 & $33.3 \%$ \\
\hline 40 o más & 15 & $45.5 \%$ \\
\hline \multicolumn{3}{|l|}{ Sexo } \\
\hline Femenino & 5 & $15.2 \%$ \\
\hline Masculino & 28 & 84.8 \\
\hline \multicolumn{3}{|l|}{ Estado Civil } \\
\hline Soltero & 12 & $36.4 \%$ \\
\hline Casado & 14 & $42.4 \%$ \\
\hline Divorciado & 6 & $18.2 \%$ \\
\hline $\begin{array}{l}\text { Otro } \\
\text { Educación (en años) }\end{array}$ & 1 & $3.0 \%$ \\
\hline Hasta 6 años & 3 & $9.1 \%$ \\
\hline 7 a 9 años & 11 & $33.3 \%$ \\
\hline 10 a 12 años & 9 & $27.3 \%$ \\
\hline 13 o más & 10 & $30.3 \%$ \\
\hline \multicolumn{3}{|l|}{ Ocupación } \\
\hline Hogar & 2 & $6.1 \%$ \\
\hline Estudiante & 2 & $6.1 \%$ \\
\hline Con empleo Estable & 9 & $27.3 \%$ \\
\hline Desempleado & 20 & $60.5 \%$ \\
\hline \multicolumn{3}{|l|}{ Estatus Socioeconómico } \\
\hline Bajo & 4 & $12.1 \%$ \\
\hline Medio Bajo & 18 & $54.6 \%$ \\
\hline Medio & 7 & $21.2 \%$ \\
\hline Medio Alto & 4 & $12.1 \%$ \\
\hline \multicolumn{3}{|l|}{ Sustancia de mayor impacto } \\
\hline Alcohol & 10 & $30.3 \%$ \\
\hline Marihuana & 8 & $24.2 \%$ \\
\hline Solventes & 1 & $3.0 \%$ \\
\hline Crystal & 6 & $18.2 \%$ \\
\hline Crack & 3 & $9.1 \%$ \\
\hline Cocaína & 5 & $15.2 \%$ \\
\hline \multicolumn{3}{|l|}{ Diagnóstico del Eje I } \\
\hline Dependencia a Sustancias & 25 & $75.7 \%$ \\
\hline Otros Trastornos & 8 & $24.3 \%$ \\
\hline
\end{tabular}

El 24.2\% no presentó trastorno de la personalidad, de éste porcentaje el $6.1 \%$ dio resultado negativo al examen de detección de sustancias en orina y el $18.2 \%$ no asistió a la cita. El 36.4\% presentó trastorno de personalidad, de los cuales $15.2 \%$ 
no asistió al examen de detección de sustancias y el 21.2\% de los pacientes reportó haber consumido, al contactarlos en el seguimiento telefónico; el 39.4\% mostró rasgos de personalidad de los grupos $\mathrm{A}, \mathrm{B}$ y $\mathrm{C}$, de éste porcentaje el $18.2 \%$ no asistió al examen de detección de sustancias en orina y el 15.2\% si asistió y obtuvo resultado negativo, el $6.1 \%$ restante en el seguimiento telefónico reportó haber consumido sustancias, $\mathrm{x}^{2}(4, N=33)=12.67, p=.013$.

Tabla 2

Diagnóstico de la personalidad en los participantes según entrevista SCID II

\begin{tabular}{lll}
\hline Diagnóstico & Frecuencia & Porcentaje \\
\hline No presento Diagnóstico & 8 & $24.2 \%$ \\
Trastorno Antisocial de la Personalidad & 9 & $27 . \%$ \\
Trastorno Obsesivo Compulsivo de la Personalidad & 1 & $3.0 \%$ \\
Trastorno Paranoide de la Personalidad & 1 & $3.0 \%$ \\
Trastorno Limítrofe de la Personalidad & 1 & $3.0 \%$ \\
Rasgo de la Personalidad del Grupo A & 4 & $12.1 \%$ \\
Rasgo de la Personalidad del Grupo B & 6 & $18.2 \%$ \\
Rasgo de la Personalidad del Grupo C & 3 & $9.2 \%$ \\
TOTAL & 33 & $100 \%$ \\
\hline
\end{tabular}

Tabla 3

Diagnóstico la entrevista SCID II y resultados al seguimiento en la muestra ( $n=33)$

\begin{tabular}{lcccc}
\hline Diagnóstico & $\begin{array}{c}\text { Inasistencia al } \\
\text { examen }\end{array}$ & $\begin{array}{c}\text { Resultado } \\
\text { Negativo }\end{array}$ & $\begin{array}{c}\text { Reporte Telefónico } \\
\text { de Consumo }\end{array}$ & Total \\
\hline Sin Diagnóstico & 6 & 2 & 0 & 8 \\
& $18.2 \%$ & $6.1 \%$ & $0.0 \%$ & $24.2 \%$ \\
Trastorno de & 5 & 0 & 7 & 12 \\
Personalidad & $15.2 \%$ & $0.0 \%$ & $21.2 \%$ & $36.4 \%$ \\
Rasgos de & 6 & 5 & 2 & 13 \\
Personalidad & $18.2 \%$ & $15.2 \%$ & $6.1 \%$ & $39.4 \%$ \\
Total & 17 & 7 & 9 & 33 \\
& $51.5 \%$ & $21.2 \%$ & $27.3 \%$ & $100.0 \%$ \\
\hline
\end{tabular}

Prueba Chi Cuadrado $\mathrm{p}=.013$

\section{DISCUSIÓN}

El hallazgo más importante de este trabajo sigue la línea de la hipótesis planteada, pues los pacientes con trastorno de la personalidad presentaron recaídas posttratamiento en el período de seguimiento a cuatro meses. Los pacientes sin ningún diagnóstico o que sólo presentaban rasgos y que asistieron al examen obtuvieron resultado negativo. 
La prevalencia de trastornos de personalidad fue del $36.4 \%$, que está dentro del margen encontrado por López \& Becoña (2006). El trastorno más prevalente fue el antisocial, como coincide en otros estudios (De Medina, 2008; Pedrero et al, 2013) y se encontraron como secundarios el trastorno limítrofe y el obsesivo compulsivo, que aparecen también en la literatura (Martínez et al., 2016; Pedrero et al., 2013). Sin embargo, el $40 \%$ de los pacientes presentó rasgos de la personalidad de cualquiera de los tres grupos de la clasificación del DSM-IV. Resulta llamativo que en el único estudio que utilizó la entrevista SCID-II, la prevalencia de trastornos fue muy alta (90\%) pero podrían considerarse diferencias como el tamaño de la muestra o ciertos aspectos culturales por reunir a participantes de varios orígenes distintos (Bricolo et al., 2002).

Aunque los trastornos de la personalidad no son la única variable asociada a la recaída y falta de adherencia al tratamiento en estos pacientes (MartínezGonzález, 2014; Nonnis, 2008; Romero, Pedrero \& Pérez, 2007), los resultados sugieren que la inclusión de un tratamiento específico para trastornos de la personalidad en los consumidores de sustancias podría ser de gran ayuda, dada su alta asociación con la recaída temprana.

Los aportes de enfoques como el psicoanálisis en la explicación de estos trastornos (de (Braier \& Guisen, 2012; Clarkin, Yeomans \& Kernberg, 2007, de Cortés, 2000; Kernberg, 1984; Rodríguez Sutil, 2005) y sus recientes desarrollos terapéuticos como la terapia de mentalización (Gergely, Fonagy, Jurist \& Target, 2002; Kernberg, 2014) han permitido incidir en los rasgos propios de cada uno de los trastornos y en conductas como impulsividad, autodestrucción, identidad difusa, oscilaciones marcadas en preferencias y estados de ánimo, relaciones caóticas y consumo de sustancias (Kernberg, 2014; Otiñano, Ponce, Fornós y Laín, 2015, Reneses et al., 2013). Por otro lado, el enfoque cognitivo-conductual (Beck, Rush, Shaw \& Emery, 1983) ha hecho grandes aportaciones en el tratamiento de los síndromes clínicos del Eje I del DSM-IV (APA, 2003), pero dado que éstos están constituidos de una manera cualitativamente distinta que los trastornos de la personalidad, el término "esquema" (Clark, Beck \& Alford, 1999) ha permitido desarrollar abordajes con la precisión que requieren estos trastornos tan complejos. De manera más reciente, Young (2003) introdujo el término "Esquema Temprano Maladaptativo" (ETM) como un factor común a los trastornos de la personalidad y se ha desarrollado la "Terapia de Esquemas", que ha mostrado su viabilidad para el tratamiento de los trastornos de la personalidad y otros comórbidos como el consumo de sustancias (Suárez y Valdiviezo, 2015). Sin embargo, el período de intervención terapéutica debe ser considerado a mediano y largo plazo, lo que no siempre es asequible en los tratamientos ofertados para adicciones.

Por último, entre las limitaciones de este estudio se encuentran el reducido tamaño de la muestra, el alto índice de pérdida de pacientes en el seguimiento, que ocurrió en parte debido a sus características de personalidad y en parte a 
sus condiciones vitales (residencia, trabajo), lo que impidió tener un reporte más completo en el seguimiento. Entre las fortalezas de este estudio está la de utilizar la entrevista SCID II, que es el instrumento basado en el DSM-IV disponible hasta el momento y que no sólo incluye el autorreporte del paciente sino la experiencia y habilidad del clínico para diagnosticar mediante la entrevista y la observación (Arias et al., 2013; Esbec y Echeburúa, 2014). Otra importante fortaleza es haber incluido el seguimiento telefónico y el examen de detección de sustancias en orina para la evaluación de la recaída o la abstinencia de los pacientes, pues ambas formas de seguimiento permitieron tener una evidencia objetiva de este aspecto de su evolución ${ }^{6}$.

\section{CONCLUSIONES}

1. Los pacientes que mostraron un trastorno de la personalidad presentaron recaídas post-tratamiento en el período de seguimiento a cuatro meses.

2. El trastorno más prevalente fue el antisocial y coincide en otros estudios; un porcentaje elevado de pacientes presentó rasgos de la personalidad de los tres grupos de la clasificación.

3. Es necesaria la inclusión de estrategias terapéuticas enfocadas a atender los trastornos de la personalidad, que frecuentemente los consumidores de sustancias padecen, dada la alta asociación con la recaída temprana.

\section{REFERENCIAS}

American Bio Medical Corporation (2015). Rapid Drug Screening. Quick Reference Guide. file://C:/Users/Usuario/Downloads/QRG_RapidDrugScreeninserto\%20 $-2015 \% 20(1)$.pdf (material informativo proporcionado por Devor Diagnósticos, S.A de C.V).

American Psychiatric Association. (2003). DSM-IV-TR Breviario: Criterios diagnósticos. Barcelona: Masson.

Arias, F., Szerman, N., Vega, P., Mesias, B., Basurte, I., Morant, C., y Babin, F. (2013). Abuso o dependencia al cannabis y otros trastornos psiquiátricos. Estudio en Madrid sobre prevalencia de patología dual. Actas Esp Psiquiatr, 122-129.

Beck, A. T., Rush, A. J., Shaw, B. F., y Emery, G. (1983). Terapia cognitiva de la depresion. Bilbao: Desclée de Brouwer.

Benjet, C., Borges, G., \& Medina-Mora, M. E. (2008). DSM-IV personality disorders in Mexico: results from a general population survey. Revista Brasileira de Psiquiatria, 30(3), 227-234.

\footnotetext{
6. Agradecimiento al Mtro. Enrique de Jesús Aceves Arce, Coordinador Regional Centro Occidente de Centros de Integración Juvenil A.C., al Lic. Luis Gómez Villaseñor, Director de la UTRO, a la Lic. Ruth Elizabeth Carrillo López y al Mtro. Octavio César Carrillo Flores, miembros del personal de la UTRO por su valioso apoyo en el desarrollo de la evaluación; a la Dra. Brenda Pérez Méndez y de manera especial a la Lic. Lucía G. Reyes Ramos de DEVOR DIAGNÓSTICOS S.A de C.V. por la autorización, financiamiento y facilidades para practicar los exámenes de detección de sustancias en orina.
} 
Braier, E. A. (2012). La estructura limítrofe. Una perspectiva teórico-clínica desde la metapsicología freudiana. Revista de psicoanálisis, 67, 129-163.

Bricolo, F., Gomma, M., Bertani, M. E., y Serpelloni, G. (2002). Prevalencia de trastornos de personalidad en una muestra de 115 clientes con trastornos por uso de drogas. Adicciones, 14(4), 491-496.

Clark, D. A., Beck, A. T., \& Alford, B. A. (1999). Scientific foundations of cognitive theory and therapy of depression. New York: John Wiley and Sons.

Clarkin, J. F., Yeomans, F. E., \& Kernberg, O. F. (2007). Psychotherapy for borderline personality: Focusing on object relations. American Psychiatric Pub. Capítulo 1 The Nature of Borderline Organization.

Corona-Figueroa, B. A., Robles García, R., Meda-Lara, R. M., Fresán Orellana, A., y Cruz-Maycott, J. A. H. (2015). Competencia y compartimiento emocional en usuarios de cocaína: Psicometría y relación con variables sociodemográficas y clínicas. Salud Mental, 38(6), 403-408.

Gómez de Cortés, C. (2000). ¿Quién es el paciente limítrofe? Salud Mental, 23(1), 30-38.

De Medina, R. B., Echeburúa, E., y Aizpiri, J. (2008). Diferencias de sexo en la dependencia del alcohol: dimensiones de personalidad, características psicopatológicas y trastornos de personalidad. Psicothema, 20(2), 218-223.

Esbec, E. y Echeburúa, E. (2011). La reformulación de los trastornos de la personalidad en el DSM-V. Actas Esp Psiquiatr, 39(1), 1-11.

Esbec, E. y Echeburúa, E. (2014). La evaluación de los trastornos de la personalidad según el DSM-5: recursos y limitaciones. Terapia psicológica,32(3), 255-264.

Fernández-Montalvo, J. y Landa, N. (2003). Comorbilidad del alcoholismo con los trastornos de personalidad (Comorbidity between alcoholism and personality disorders). Clinica y Salud, 14, 27-41.

Fernández-Montalvo, J. y Javier, J. (2004). Trastornos de personalidad y abandonos terapéuticos en pacientes adictos: resultados en una comunidad terapéutica. International Journal of Clinical and Health Psychology, 4(2), 271-283.

First, M. B., Gibbon, M., Spitzer, R. L., Williams, J. B. W. \& Benjamin, L. S. (1997). User's Guide for the Structured Clinical Interview for DSM-IV Axis II Personality Disorders. Washington: American Psychiatric Press, Inc.

Fonagy, P., Gergely, G., \& Jurist, E. L. (Eds.). (2004). Affect regulation, mentalization and the development of the self. Karnac books.

García-Rodríguez, O., Secades-Villa, R., Álvarez Rodríguez, H., Rodríguez, A. R., Fernández-Hermida, J. R., Carballo, J. y Al-Halabí Díaz, S. (2007). Efecto de los incentivos sobre la retención en un tratamiento ambulatorio para adictos a la cocaína. Psicothema, 19(1), 134-139.

Herrero, J. (2004). Alteraciones de la personalidad asociadas a las conductas adictivas: influencia de la duración del consumo y sus implicaciones. Psykhe (Santiago), 13(1), 91-100. 
Kernberg, O. F. (1993). Severe personality disorders: Psychotherapeutic strategies. Yale University Press.

Kernberg, O. F. (2015). Neurobiological correlates of objectrelations theory: The relationship between neurobiological and psychodynamic development. International Forum of Psychoanalysis, 24(1), 38-46.

López, A., \& Becoña, E. (2006). Dependencia de la cocaína y psicopatología a través del SCL-90-R. Revista de Psicopatología y Psicología Clínica, 11(1), 13-20.

Martínez-González, J. M., Albein-Urios, N., Lozano-Rojas, O. y Verdejo-García, A. (2014). Aspectos diferenciales del riesgo de abandono al inicio del tratamiento de la adicción a la cocaína en pacientes con trastornos de la personalidad. Adicciones, 26(2), 116 125.

Martínez-González, J. M., Graña, J. L. y Trujillo, H. M. (2009). Influencia de los trastornos de la personalidad y patrones de consumo en la eficacia de un programa de prevención de recaídas para el tratamiento del alcoholismo. Adicciones, 21(2), 105-112.

Martínez González, J. M., Graña Gómez, J. L. y Trujillo Mendoza, H. (2011). Estudio longitudinal sobre calidad de vida, craving y ajuste psicológico en pacientes dependientes del alcohol: variaciones en función de los trastornos de la personalidad. Adicciones, 23(3), 227-235.

Martínez-González, J. M., Vilar López, R., Becoña Iglesias, E. y Verdejo-García, A. (2016). Self-deception as a mechanism for the maintenance of drug addiction. Psicothema, 28(1).

Nonnis, B. N. T. (2008). Una Trimorbilidad Forense Emergente: El Trastorno de Personalidad, el Trastorno del Control de los Impulsos y el Abuso de Sustancias. Anuario de Psicología Jurídica, 18, 91-97.

Otiñano, M. I., Ponce, I. M., Fornós, G. M. y Laín, M. M. (2015). Comportamiento adictivo e inestabilidad emocional: a propósito de un caso. Cuadernos de medicina psicosomática y psiquiatría de enlace, (115), 32-40.

Pedrero Pérez, E. J., Puerta García, C., Lagares Roibas, A. y Sáez Maldonado, A. (2003). Prevalencia e intensidad de los trastornos de personalidad en adictos a sustancias en tratamiento en un centro de atención a las drogodependencias. Trastornos Adictivos, 5(3), 241-255.

Pedrero Pérez, E. J., \& Segura López, I. (2003). Los trastornos de la personalidad en drogodependientes y su relación con la dificultad de manejo clínico. Trastornos adictivos, 5(3), 229-240.

Pedrero-Pérez, E. J., Ruiz-Sánchez de León, J. M., Lozoya-Delgado, P., Rojo-Mota, G., Llanero-Luque, M. y Puerta-García, C. (2013). Sintomatología prefrontal y trastornos de la personalidad en adictos a sustancias. Revista de Neurología, 56(4), 205-213.

Reneses, B., Galián, M., Serrano, R., Figuera, D., Fernández Del Moral, A., López-Ibor, J. J. y Trujillo, M. (2013). Una nueva Psicoterapia Breve para Trastornos límite de la personalidad. Resultados preliminares de un Ensayo controlado y aleatorizado. Actas Españolas de Psiquiatría, 41(3), 139-148. 
Rodríguez Sutil, C. (2005). El concepto de" carácter" en psicoanálisis. Sobre una patología sin síntomas. Intersubjetivo, 1(7), 5-27.

Romero, J. C., Pérez, E. J. P., y López, M. P. (2007). Autoeficacia para resistirse al consumo de sustancias como predictora de resultados de tratamiento y su relación con variables de personalidad: estudio de una muestra de adictos con el DTCQ, el VIP y el MCMI-II. Adicciones, 19(2), 141-152.

Sánchez Huesca, R., Guisa Cruz, V. M., Cedillo González, A. y Pascual Blanco, Y. (2002). Perfil de personalidad en usuarios de cocaína utilizando el Inventario Multifásico de Personalidad. Actas Españolas de Psiquiatría, 30(2), 91-98.

Sánchez, R. (2003). Theodore Millon, una teoría de la personalidad y su patología. PsicoUSF, 8(2), 163-173.

Suarez, J. E. y Valdiviezo, A. F. (2015) Terapia de esquemas en el consumo de alcohol de adolescentes de la unidad educativa del milenio penipe, periodo Diciembre 2014Mayo 2015. (Tesis de pregrado) Universidad Nacional de Chimborazo, Riobamba, Ecuador.

Young, J. E., Klosko, J. S., \& Weishaar, M. E. (2003). Schema therapy: A practitioner's guide. New York, NY: Guilford Press. 
